\title{
Comparison of real world and core laboratory lupus anticoagulant results from the Antiphospholipid Syndrome Alliance for Clinical Trials and International Networking (APS ACTION) clinical database and repository
}

\author{
Maria Efthymiou ${ }^{1}$ (D) | lan J. Mackie ${ }^{1}$ (D) | Philip J. Lane ${ }^{1}$ (D) | Danieli Andrade ${ }^{2}$ | \\ Rohan Willis $^{3}$ (D) | Doruk Erkan ${ }^{4}$ (D) | Savino Sciascia5 ${ }^{5}$ | Steven Krillis ${ }^{6}$ | Elisa Bison ${ }^{7}$ | \\ Margarete Borges Galhardo Vendramini ${ }^{2}$ | Zurina Romay-Penabad ${ }^{3}$ | Miao Qi ${ }^{6}$ | \\ Maria Tektonidou $^{8}$ (D) | Amaia Ugarte ${ }^{9}$ | Cecilia Chighizola ${ }^{10}$ | H. Michael Belmont ${ }^{11}$ | \\ Maria Angeles Aguirre ${ }^{12}$ | Lanlan $\mathrm{Ji}^{13}$ | D. Ware Branch ${ }^{14}$ | Guilherme de Jesus ${ }^{15}$ | \\ Paul R. Fortin ${ }^{16}$ | Laura Andreoli ${ }^{17,18}$ | Michelle Petri $^{19}$ | Ricard Cervera ${ }^{20}$ (D) | \\ Esther Rodriguez $^{21}$ | Jason S. Knight ${ }^{22}$ (D) | Tatsuya Atsumi ${ }^{23}$ (D) | Joann Vega ${ }^{7}$ | \\ Ecem Sevim ${ }^{7}$ (D) | Maria Laura Bertolaccini ${ }^{24}$ (D) | Vittorio Pengo ${ }^{6}$ (D) | \\ Hannah Cohen ${ }^{1,25}$ (D) | on behalf of APS ACTION† \\ ${ }^{1}$ Haemostasis Research Unit, Department of Haematology, University College London, London, UK \\ ${ }^{2}$ University of Sao Paulo, Sao Paulo, Brazil \\ ${ }^{3}$ Antiphospholipid Standardization Laboratory, University of Texas Medical Branch, Galveston, TX, USA \\ ${ }^{4}$ Barbara Volcker Center for Women and Rheumatic Diseases, Hospital for Special Surgery, Weill Cornell Medicine, New York, NY, USA \\ ${ }^{5}$ Center of Research of Immunopathology and Rare Diseases, University of Turin, Turin, Italy \\ ${ }^{6}$ Department of Infectious Diseases, Immunology and Sexual Health, St George Hospital, Sydney, NSW, Australia \\ ${ }^{7}$ University Hospital Padova, Padova, Italy \\ ${ }^{8}$ National and Kapodistrian University of Athens, Athens, Greece \\ ${ }^{9}$ Internal Medicine, Hospital Universitario Cruces, Barakaldo, País Vasco, Spain \\ ${ }^{10}$ University of Milan, Milan, Italy \\ ${ }^{11}$ School of Medicine, New York University, New York, NY, USA \\ ${ }^{12}$ Maimonides Institute for Biomedical Research of Cordoba, Cordoba, Spain \\ ${ }^{13}$ Rheumatology and Immunology Department, Peking University First Hospital, Beijing, China \\ ${ }^{14}$ University of Utah and Intermountain Healthcare, Salt Lake City, UT, USA \\ ${ }^{15}$ Rio de Janeiro State University, Rio de Janeiro, Brazil \\ ${ }^{16} \mathrm{CHU}$ de Quebec - Université Laval, Quebec, QC, Canada \\ ${ }^{17}$ Rheumatology and Clinical Immunology, Spedali Civili, Brescia, Italy \\ ${ }^{18}$ Department of Clinical and Experimental Sciences, University of Brescia, Brescia, Italy \\ ${ }^{19}$ Department of Medicine, Johns Hopkins University School of Medicine, Baltimore, MD, USA \\ ${ }^{20}$ Department of Autoimmune Diseases, Hospital Clínic Institut d'Investigacions Biomèdiques August Pi i Sunyer (IDIBAPS), Barcelona, Catalonia, Spain \\ ${ }^{21}$ Hospital Universitario 12 de Octubre, Madrid, Spain
}


${ }^{22}$ Division of Rheumatology, University of Michigan, Ann Arbor, MI, USA

${ }^{23}$ Hokkaido University Hospital, Sapporo, Japan

${ }^{24}$ Academic Department of Vascular Surgery, School of Cardiovascular Medicine \& Sciences, King's College London, London, UK

${ }^{25}$ Department of Haematology, University College London Hospitals NHS Foundation Trust, London, UK

\section{Correspondence}

Maria Efthymiou, Haemostasis Research Unit, Department of Haematology,

University College London, 1st Floor, 51 Chenies Mews, London WC1E 6HX, UK.

Email: m.efthymiou@ucl.ac.uk

\begin{abstract}
Background: Variability remains a challenge in lupus anticoagulant (LA) testing. Objective: To validate LA test performance between Antiphospholipid Syndrome Alliance for Clinical Trials and International Networking (APS ACTION) Core laboratories and examine agreement in LA status between Core and local/hospital laboratories contributing patients to this prospective registry.

Methods: Five Core laboratories used the same reagents, analyzer type, protocols, and characterized samples for LA validation. Non-anticoagulated registry samples were retested at the corresponding regional Core laboratories and anticoagulated samples at a single Core laboratory. Categorical agreement and discrepancies in LA status between Core and local/hospital laboratories were analyzed.

Results: Clotting times for the reference/characterized plasmas used for normalized ratios were similar between Core laboratories ( $C V<4 \%$ ); precision and agreement for LA positive/negative plasma were similar (all $\mathrm{CV} \leq 5 \%$ ) in the four laboratories that completed both parts of the validation exercise; 418 registry samples underwent LA testing. Agreement for LA positive/negative status between Core and local/hospital laboratories was observed in 87\% (115/132) non-anticoagulated and 77\% (183/237) anticoagulated samples. However, $28.7 \%$ (120/418) of samples showed discordance between the Core and local/hospital laboratories or equivocal LA results. Some of the results of the local/hospital laboratories might have been unreliable in $24.7 \%(41 / 166)$ and $23 \%(58 / 252)$ of the total non-anticoagulated and anticoagulated samples, respectively. Equivocal results by the Core laboratory might have also contributed to discordance. Conclusions: Laboratories can achieve good agreement in LA performance by use of the same reagents, analyzer type, and protocols. The standardized Core laboratory results underpin accurate interpretation of APS ACTION clinical data.
\end{abstract}

\section{KEYWORDS}

antiphospholipid antibodies, antiphospholipid syndrome, lupus anticoagulant, Russell's viper venom time, thrombosis

\section{1 | INTRODUCTION}

Antiphospholipid syndrome (APS) is a potentially life-threatening acquired autoimmune disorder manifested by thrombosis (arterial, venous, or microvascular) and/or obstetric morbidity in association with persistently positive antiphospholipid antibodies ( $\mathrm{aPL}$ ), ie lupus anticoagulant (LA), IgG and/or IgM anticardiolipin ( $\mathrm{aCL}$ ), and/ or anti-beta 2 glycoprotein I antibodies (aß2GPI). ${ }^{1}$ Accurate laboratory diagnosis of APS is crucial as these patients inherently differ from other patients with similar clinical features. In particular, the presence of $\mathrm{aPL}$ is associated with an increased risk of recurrent thrombosis and hence these patients are candidates for long-term

\section{Essentials}

- Variability remains a challenge in lupus anticoagulant (LA) testing.

- Good agreement in LA performance achieved by use of the same reagents, analyser type and protocols.

- Local/hospital laboratory LA results might have been unreliable in $\sim 24 \%$ of all samples.

- Standardized LA testing is required to underpin accurate interpretation of clinical data. 
anticoagulation. $^{2}$ Detection of LA is problematic because of its heterogeneity and the variable sensitivity and specificity of different LA tests. False-positive or false-negative LA status may be detrimental to patient management as it could lead to unnecessary anticoagulation with the risk of bleeding, or inadequate thromboprophylaxis and risk of thrombosis recurrence. Detection of LA in plasmas from anticoagulated patients is especially problematic because of their prolonged clotting times. Recommendations published in 2009 by the International Society of Thrombosis and Haemostasis Scientific Standardisation Subcommittee (ISTH SSC) on Lupus Anticoagulant/Phospholipid Antibodies for the detection of LA, as well as the British Society of Haematology (BSH) (2012) and Clinical \& Laboratory Standards Institute (CLSI) guidelines (2014) have proven to be useful guidance on how to perform LA tests. LA detection should be a three-step procedure, comprising a screening step with an LA-sensitive phospholipid, a mixing step with normal plasma to demonstrate an inhibitor, and a confirmatory step to show phospholipid dependence. ${ }^{3-5}$

The AntiPhospholipid Syndrome Alliance For Clinical Trials and InternatiOnal Networking (APS ACTION) is an international network created specifically to design and conduct well-designed, large-scale, multi-center studies in persistently aPL-positive patients. ${ }^{6}$ Since 2010 , APS ACTION has been conducting a 10 -year international clinical database and blood sample repository ("registry") to study the disease course in aPL-positive patients with/without other systemic autoimmune diseases. The network includes 50 physician-scientists from 25 international centers. APS ACTION registry laboratory inclusion criteria are moderate/high titer $\mathrm{aCL}$ and/or a $32 \mathrm{GPI}$ and/or LA test based on the International consensus criteria, ${ }^{1}$ tested at least twice 12 weeks apart, within 1 year prior to enrolment. A secure web-based data capture system (REDCap) is used to store patient information. Blood samples and clinical information are collected from patients at inclusion (baseline) and then annually. APS ACTION has sought to standardize aPL laboratory testing that is of paramount importance to improve the interpretation and reliability of laboratory tests for associated clinical trials and research studies. Five APS ACTION Core laboratories were initially set up worldwide, in Sao Paulo (Brazil), Sydney (Australia), Galveston (USA), Padova (Italy), and London (UK), to store blood samples from hospitals in their continent/geographic region. Each Core laboratory performs aPL tests using standard protocols and reagents to confirm the original hospital result.

The aims of our study were to: (a) validate the LA test performance between the five APS ACTION Core laboratories; and (b) examine the degree of agreement in LA status between Core and local/ hospital laboratories contributing patients to the registry.

\section{2 | METHODS}

\section{1 | Validation of Core laboratories' LA test performance}

All five Core laboratories used an ACL TOP500 analyzer (Werfen) and performed the dilute Russell's viper venom time (DRVVT) and silica clotting time (SCT) with the same Lot numbers of HemosIL DRVVT Screen/Confirm and HemosIL silica clotting time (SCT) (SCT Screen and SCT Confirm reagents). All laboratories were provided and followed a common study protocol and laboratory manual for LA testing designed specifically for APS ACTION. All laboratories used the same set of characterized plasmas for the validation exercise to establish agreement in LA testing between the Core laboratories as follows: 1st International Reference Panel for LA (National Institute for Biological Standards and Control [NIBSC], South Mimms, UK; comprising: LA negative [NLA], moderate positive [MLA], and strong positive [SLA] LA samples), as well as HemosIL LA negative (LA-) \& HemosIL LA positive (LA+) quality control plasmas (QC), and these were tested on each of three working days. The manufacturer's cutoff values for all tests were validated in each Core laboratory using plasmas from at least 40 healthy normal subjects prior to any tests being performed, according to national and international guidelines. ${ }^{4,7}$ The results were calculated as normalized ratios, using local pooled normal plasma, or where this was not available, a commercial pool or LA negative control plasma. The within- and between-center precision was investigated for each plasma. The five laboratories were anonymized as A-E in no particular order.

\subsection{Assessment of agreement in LA status between Core and local/hospital laboratories}

Each Core laboratory assessed LA status in samples collected from their allocated local/hospital laboratories within the first year of entry into the registry. All laboratories followed the APS ACTION protocol for preparation of samples, storage, and LA testing. Samples were processed within four hours of collection and platelet poor plasma (PPP) was prepared from blood withdrawn by venipuncture in $0.109 \mathrm{~mol} / \mathrm{L}$ sodium citrate $9: 1$, then double-centrifuged at $1500 \mathrm{~g}$ for 15 minutes. To reduce variability, samples were stored at $-80^{\circ} \mathrm{C}$ immediately after preparation and shipped on dry ice as this has been reported to cause minimum interference with LA testing and analysis. ${ }^{8,9}$ Samples were thawed uncapped at $37^{\circ} \mathrm{C}$ (using a water bath) prior to assessment as recommended by CLSI. ${ }^{5}$ Samples from non-anticoagulated patients $(n=166)$ were assessed using DRVVT and SCT reagents, as detailed above, and an ACL TOP500 analyzer. Equal volume mixtures of patient and normal plasma were tested to confirm the presence of an inhibitor.

Lupus anticoagulant status for patients receiving anticoagulation was assessed at a single Core laboratory (UK). For patients receiving vitamin $\mathrm{K}$ antagonists (VKA), the DRVVT was performed with Screen and Confirm reagents on equal volume mixtures of patient/normal plasma (which if positive confirmed the presence of an inhibitor and phospholipid dependence); and the Taipan venom time (TVT)/Ecarin clotting time (ECT; Diagnostic Reagents Ltd) ratio was performed on undiluted patient samples as well as on equal volume mixtures of patient/normal plasma if the TVT was prolonged. The SCT Screen and Confirm tests on equal volumes of patient/normal plasma was not performed in patients receiving VKA due to the limited volume of plasma available. Patients 
dRVVT Screen - LA+QC Plasma

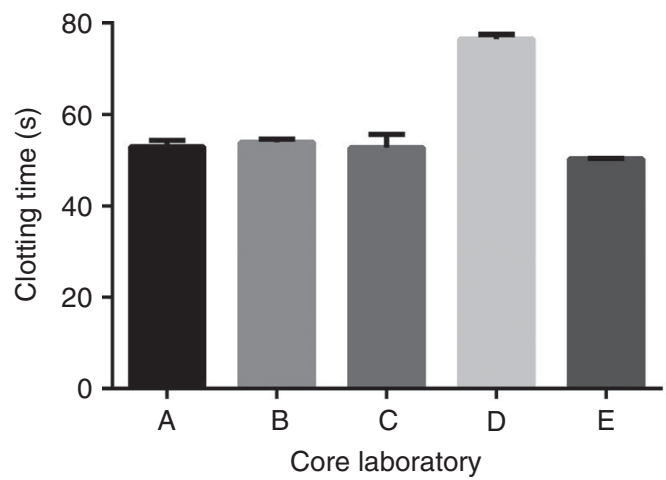

dRVVT Screen - SLA Plasma

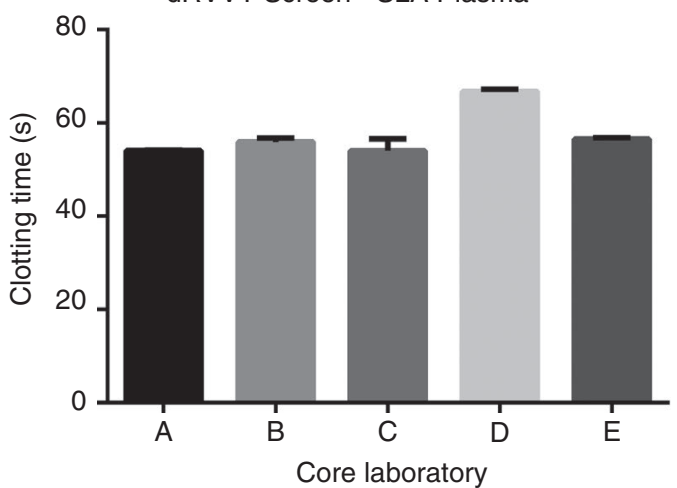

dRVVT Screen - LA+QC Plasma

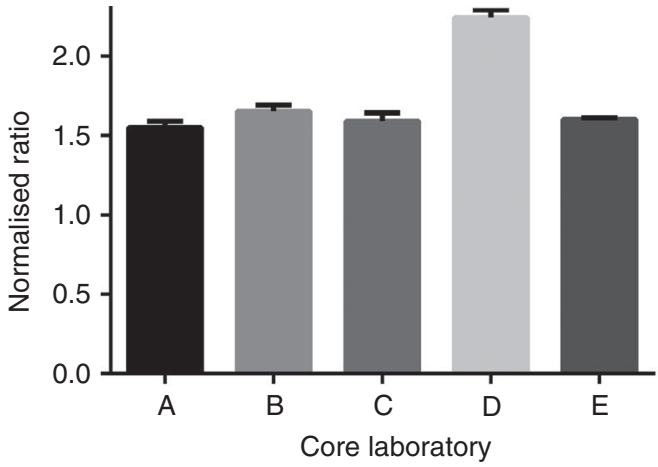

dRVVT Screen - SLA Plasma

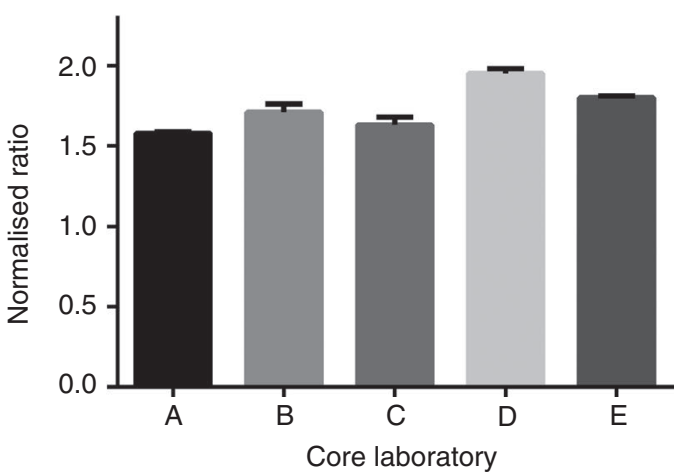

FIGURE 1 Dilute Russell's viper venom time (DRVVT) Screen test used with lupus anticoagulant (LA) positive (+) quality control (QC) plasma and for strong positive LA (SLA) plasma: All five Core laboratories used an ACL TOP500 analyzer and performed the DRVVT with the same lot numbers of HemosIL DRVVT Screen/Confirm. All labs were provided with a study protocol and laboratory manual for LA testing. Results are shown for clotting times and normalized ratios for the fresh vial of the 1st International Reference Panel SLA plasma sample and the HemosIL LA+ QC plasma, tested on each of three working days. The results were calculated as normalized ratios, using local pooled normal plasma, or where this was not available, a commercial pool or LA negative control plasma

receiving low molecular weight heparin $(\mathrm{LMWH})$ prophylaxis were assessed by DRVVT (Screen/Confirm) and SCT (DRVVT reagents are insensitive to heparin or $\mathrm{LMWH}$ up to $1.0 \mathrm{IU} / \mathrm{mL}$; SCT reagents are insensitive to heparin up to $0.5 \mathrm{IU} / \mathrm{mL}$ and $\mathrm{LMWH}$ to $1.0 \mathrm{IU} /$ $\mathrm{mL}$ ). For patients receiving rivaroxaban, a direct oral anticoagulant (DOAC) factor (F) Xa inhibitor, LA status was assessed by TVT/ ECT ratio and rivaroxaban levels were measured with an anti-FXa amidolytic assay using specific calibrators.

Lupus anticoagulant status was considered positive if the patient/normal DRVVT or SCT Screen ratio was $\geq 1.20$ and the Screen/ Confirm normalized ratio was $\geq 1.20$. TVT/ECT was considered positive if the TVT was prolonged and the normalized TVT/ECT ratio was $>1.20$. The TVT/ECT ratio cut-off of $1.20^{10}$ was established locally in accordance with CLSI guidelines. ${ }^{7}$

In all tests, evidence of inhibition was provided by testing equal volume mixtures of patient and normal plasma. Mixing tests were interpreted as indicating an inhibitor if the test/normal ratio was greater than the cut-off for normal plasma. Results were recorded as equivocal if a mixing test was not performed (due to insufficient plasma volume) or if an inhibitor was not demonstrated, and the test/confirm ratio was positive. Results were reported as positive, negative ("not detected" in the case of anticoagulated patients), or equivocal (where there was no evidence of an inhibitor or suspicion of an underlying coagulopathy).

\section{3 | Statistical analysis}

Agreement of categorical positive and negative aPL test results was assessed using $\kappa$-coefficients (<.20, poor; .21-.40 fair; .41-.60 moderate; $.61-.80$ good; $.81-1.00$ very good) ${ }^{11}$ and with the Holley and Gilford's $G$ test with a $99 \%$ confidence as our results were skewed. The $G$-test is equivalent to several other inter-rater agreement statistics that have been proposed over the years. In contrast to kCohen, the calculation of chance agreement in $\mathrm{G}$ does not depend on the obtained frequencies but is defined a priori. The $G$-test statistic is a measure of how much overall variation there is from an ideal prediction that you would expect if all versions were the same. Even if there is no hard rule, however, a common benchmark is $99 \%$ confidence, meaning that with the obtained G-test values, we have less than a $1 \%$ chance of making a mistake at the point in time where we made our decision on test agreement. ${ }^{12}$ All statistical analysis was performed using SPSS 19.0 (IBM Software). 
TAB LE 1 Normalised Screen/Confirm ratios in each Core laboratory for DRVVT and SCT: All Core laboratories identified correctly the LA status of the LA+ QC, NLA, MLA, and SLA samples (average values over three days \pm standard deviation are shown, normal cut-off taken as 1.20). Laboratory D did not return results for SCT. The LA-negative QC plasma results are not included as it mirrored the results indicated by the NLA

\begin{tabular}{|c|c|c|c|c|}
\hline DRVVT & $\mathrm{LA}+\mathrm{QC}$ & NLA & MLA & SLA \\
\hline A & $1.49 \pm 0.02$ & $0.90 \pm 0.02$ & $1.31 \pm 0.02$ & $1.51 \pm 0.02$ \\
\hline C & $1.58 \pm 0.05$ & $1.01 \pm 0.02$ & $1.36 \pm 0.04$ & $1.57 \pm 0.05$ \\
\hline D & $2.04 \pm 0.05$ & $0.95 \pm 0.01$ & $1.50 \pm 0.01$ & $1.86 \pm 0.03$ \\
\hline SCT & $\mathrm{LA}+\mathrm{QC}$ & NLA & MLA & SLA \\
\hline A & $2.30 \pm 0.13$ & $0.90 \pm 0.04$ & $1.85 \pm 0.03$ & $2.21 \pm 0.12$ \\
\hline B & $2.41 \pm 0.07$ & $0.92 \pm 0.01$ & $1.84 \pm 0.03$ & $2.25 \pm 0.02$ \\
\hline C & $2.40 \pm 0.09$ & $0.92 \pm 0.01$ & $1.88 \pm 0.06$ & $2.19 \pm 0.06$ \\
\hline
\end{tabular}

Abbreviations: DRVVT, dilute Russell's viper venom time; LA, lupus anticoagulant; LA+QC, LA positive quality control plasma; MLA, moderate positive LA sample; NLA, LA negative sample; SCT, silica clotting time; SLA, strong positive LA sample.

${ }^{a}$ All replicate ratios were the same.

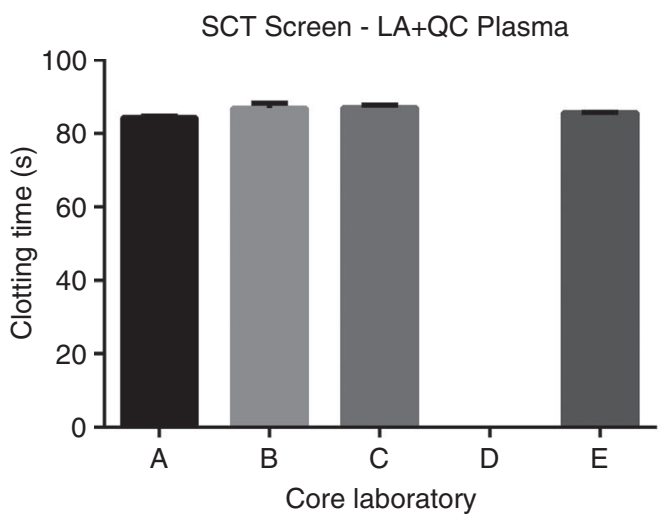

SCT Screen - SLA Plasma

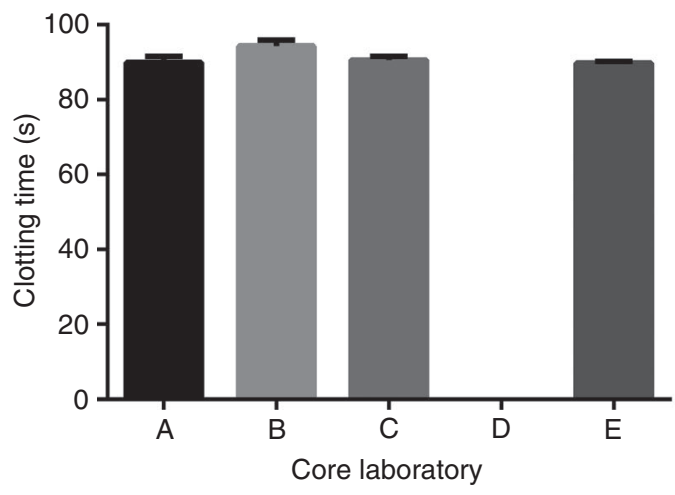

SCT Screen - LA+QC Plasma

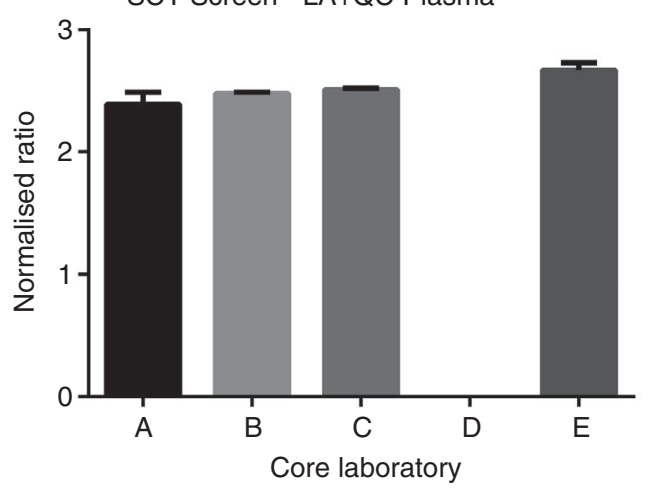

SCT Screen - SLA Plasma

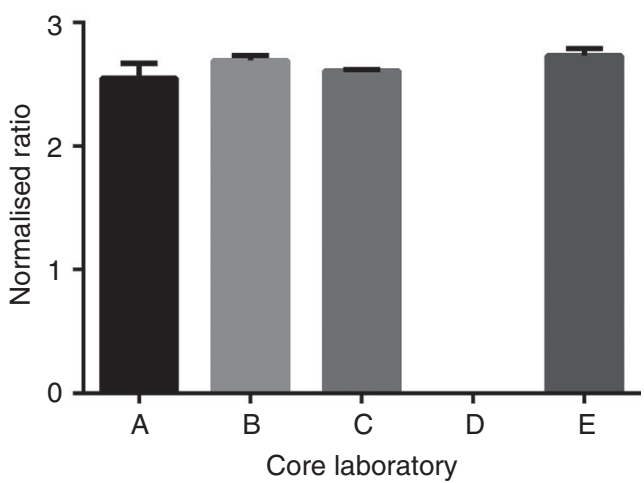

FIGURE 2 Silica clotting time (SCT) Screen test used with lupus anticoagulant (LA) positive (+) quality control (QC) plasma and for strong positive LA (SLA) plasma: All five Core laboratories used an ACL TOP500 analyzer and performed the SCT with the same Lot numbers of HemosIL SCT Screen and Confirm reagents. All laboratories were provided with a study protocol and a laboratory manual for LA testing. Results are shown for clotting times and normalized ratios for the HemosIL LA+ QC plasma and the SLA plasma sample from the 1st International Reference Panel for LA, tested on each of three working days. The results were calculated as normalized ratios, using local pooled normal plasma, or where this was not available a commercial pool or LA negative control plasma 


\section{3 | RESULTS}

\section{1 | Evaluation of LA test performance}

Two Core laboratories (B, C) used HemosIL normal control plasma (Werfen) for the calculation of normalized ratios, while three (A, D E) used in-house or commercial lyophilized (Pathway Diagnostics) or frozen (Cryocheck, Precision Biologics) pooled normal plasma. These all yielded very similar clotting times for DRVVT Screen (32.7-34.2 seconds) and Confirm (30.5-32.4 seconds), with between-laboratory coefficient of variation (CV) values $<3 \%$; and for SCT Screen (34.7-35.3 seconds) and Confirm (35.4-36.0 seconds), $\mathrm{CV}<4 \%$.

Precision and agreement were generally good between all Core laboratories for LA negative control and test plasmas using both DRVVT Screen and Confirm reagents. The between-day CV of clotting time was $<2.5 \%$ for LA negative control plasma and $<5.0 \%$ for LA positive plasmas (ie MLA \& SLA). However, one laboratory (D) tended to show longer clotting times and higher ratios for LA-positive samples (MLA \& SLA) with DRVVT Screen reagent (Figure 1), but not with Confirm reagent. Similar disparity between laboratories in clotting times and ratios was observed in mixing tests (data not shown). Despite both laboratory $\mathrm{D}$, and to a lesser extend laboratory $B$, obtaining higher average DRVVT Screen/Confirmed normalized

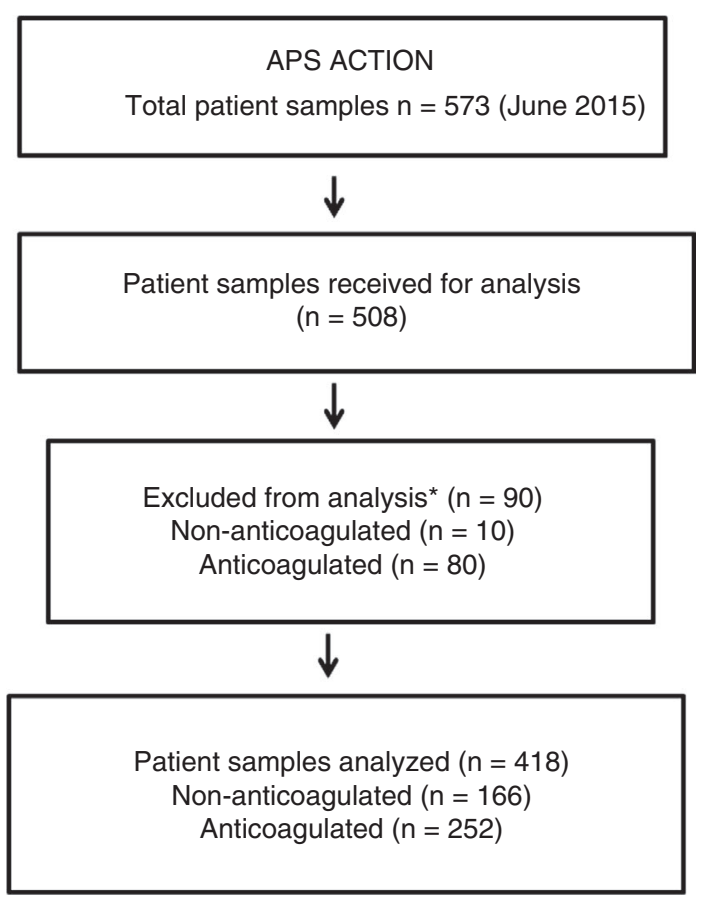

FIGURE 3 Breakdown of non-anticoagulated and anticoagulated samples received from APS ACTION, samples excluded and final numbers included in analysis: ${ }^{*}$ Ninety patient samples (10 non-anticoagulated and 80 anticoagulated) were excluded from the final analysis due to: insufficient volume of citrate plasma for further analysis $(n=38)$, or due to the local/ hospital laboratory submitting only aCL/a $\beta 2$ GPI results; or LA status not being available $(n=52)$ ratios from the other Core laboratories, all laboratories correctly identified the LA status of the LA+ QC, NLA, MLA, and SLA samples (average values over 3 days shown in Table 1).

Four Core laboratories returned results for SCT (Laboratories A, $B, C, E$ ) and showed good agreement for clotting times, with all CV values $<3.2 \%$. Although test/normal ratios for SCT Screen reagent showed some variability (Figure 2), these discrepancies disappeared when normalized Screen/Confirm ratios were calculated (Table 1). Laboratory $\mathrm{D}$ played no further role as a Core laboratory for LA assessments and did not contribute data to the results sections below.

\subsection{Agreement in LA status between Core and local/hospital laboratories}

Samples from 508 APS ACTION patients were analyzed from a total of 573 registered (until June 2015) (Figure 3). Ninety samples (10 non-anticoagulated and 80 anticoagulated) were excluded from the analysis due to: insufficient volume of citrate plasma for further analysis ( $n=38$ ), the local/hospital laboratory submitting only $\mathrm{aCL} /$ aß2GPI results, or LA status not being available $(n=52)$. Samples from the remaining 418 patients (166 non-anticoagulated and 252 anticoagulated) were analyzed (Figure 3).

Of the 418 patients analyzed, 222 (53.1\%) were diagnosed with primary APS (according to Sapporo criteria), ${ }^{1} 58$ (13.9\%) with aPL (but insufficient Sapporo criteria to fulfil APS), 86 (20.6\%) as APS with other systemic autoimmune disease, and 52 (12.4\%) had aPL without Sapporo APS criteria but with other systemic autoimmune disease. Of the 418 patients, 218 (52.2\%) were classified as thrombotic (either venous or arterial or both), 50 (12.0\%) as thrombotic with pregnancy morbidity, 40 (9.6\%) as obstetrical APS, and 110 (26.3\%) were aPL positive without APS.

For the non-anticoagulated patient samples $(n=166)$ analyzed, local/hospital laboratories submitted LA results (for the year prior to data entry): on more than three occasions in 92 patients (55.4\%), two occasions in 59 patients (31.3\%), and one occasion in 15 patients (9.0\%). Of the 252 anticoagulated patient samples analyzed, LA status was assessed and reported by the local/hospital laboratories in more than three hospital visits in 154 patients (61.1\%), two visits in 63 patients (25.0\%), one visit in 35 patients (13.9\%). Local/hospital laboratories that submitted LA results on only one occasion for the year prior to data entry had submitted either $\mathrm{aCL}$ or $\mathrm{a} \beta 2 \mathrm{GPI}$ results on more than two occasions (thus criteria for APS diagnosis had been fulfilled) before inclusion in the database.

Local/hospital laboratories were asked to record the method of LA assessment for each occasion tested (in the year prior to study entry). A total of 1039 tests were reported: 5.1\% performed activated partial thromboplastin time (aPTT)-based tests, 31.1\% DRVVT, 53.5\% aPTT-based tests and DRVVT, 8.3\% reported "unknown" and $2 \%$ as "other" (rather than DRVVT or APTT).

At inclusion in the APS ACTION registry, LA status (positive/negative) reported by the local/hospital laboratories was as follow: for non-anticoagulated samples, 70\% (111/166) LA positive and 30.0\% 
TAB LE 2 LA status of non-anticoagulated patient samples at Core and local/hospital laboratories: The number of positive and negative LA samples identified by both the laboratories and the ones that were discrepant are shown, as well as agreement and statistical results

\begin{tabular}{|c|c|c|c|c|c|c|}
\hline & & \multicolumn{5}{|c|}{ Core laboratories } \\
\hline & & POS & NEG & Agreement & K coeff & $G$-test ${ }^{a}$ \\
\hline \multirow[t]{2}{*}{ Local/hospital Laboratories } & POS & 98 & 5 & \multirow{2}{*}{$\begin{array}{l}115 / 132 \\
87.1 \%\end{array}$} & \multirow{2}{*}{$\begin{array}{l}0.589 \\
P<.001\end{array}$} & \multirow{2}{*}{$\begin{array}{l}32.6 \\
(99.8 \% \text { confidence })\end{array}$} \\
\hline & NEG & 12 & 17 & & & \\
\hline
\end{tabular}

Abbreviations: LA, lupus anticoagulant; NEG, negative; POS, positive.

${ }^{a}$ The $G$-test value as it's over $99 \%$ confidence indicates that there is $<0.2 \%$ chance of making a mistake regarding agreement of positive and negative LA results as defined.

TAB LE 3 Analysis of possible reasons for discordant and equivocal results in LA status between Core and local/hospital laboratories testing in non-anticoagulated patient samples

\begin{tabular}{|c|c|c|c|c|}
\hline \multirow{3}{*}{$\begin{array}{l}\text { Total } \\
\text { Local/hospital laboratories }\end{array}$} & \multicolumn{2}{|c|}{ Discordant } & \multicolumn{2}{|c|}{ Equivocal } \\
\hline & \multicolumn{2}{|l|}{17} & \multicolumn{2}{|l|}{34} \\
\hline & LA POS & LA NEG & LA POS & LA NEG \\
\hline $\begin{array}{l}\text { Samples tested on only one occasion at } \\
\text { local/hospital laboratory }\end{array}$ & 1 & 3 & 3 & 7 \\
\hline $\begin{array}{l}\text { Tested more than once at local/hospital } \\
\text { laboratory, but LA status varied }\end{array}$ & 2 & 4 & 4 & 6 \\
\hline $\begin{array}{l}\text { Method of LA assessment not specified or } \\
\text { only one LA method performed }\end{array}$ & 1 & 2 & 3 & 5 \\
\hline $\begin{array}{l}\text { Tested more than once at local/hospital } \\
\text { laboratory, with consistent results on LA } \\
\text { status }\end{array}$ & 1 & 3 & 3 & 3 \\
\hline
\end{tabular}

Abbreviations: LA, lupus anticoagulant; NEG, negative; POS, positive.
(50/166) LA negative and for anticoagulated samples, 84\% (211/252) LA positive and $16 \%(41 / 252)$ LA negative.

\section{3 | Non-anticoagulated patient samples}

For the 166 non-anticoagulated patients, 132 were recorded as LA positive or negative and amongst these there was $87.1 \%$ agreement between the Core and the local/hospital laboratories about LA classification ( $\mathrm{K}$ coefficient $=0.589, P<.001$; G-test statistic $32.6>99 \%$ confidence, Table 2 ). Seventeen samples (12.9\%) were discordant between the two with 12 samples identified as LA negative in the local/hospital laboratory, but positive in the Core laboratories, while five samples showed discordance in the opposite way (Table 2).

We examined possible reasons for the discordance in LA status between Core and local/hospital laboratories in these 17 patient samples. Four were only tested on one occasion at the local/hospital laboratory; six were tested more than once, but the LA status varied; in three the LA method was not specified, or only one LA method was performed (because two were recorded as LA negative, there could have been underdetection of LA). Only four samples out of the 17 discordant were tested on more than one occasion with consistent results ( 1 positive and 3 negative) (Table 3 ).

The results in 34 further samples $(20.5 \%, 34 / 166)$ were considered to be equivocal by the Core laboratories because an inhibitor could not be demonstrated. Of these, 21 had been recorded as negative and 13 as positive by the local/hospital laboratory
(Table 3). Ten of these patients only had an LA assessment on one occasion prior to inclusion in the registry. Another 10 were tested more than once at the local/hospital laboratory, but results were variable. In eight patients, either the method of LA assessment was not specified or only one LA method was performed (because five of these were recorded as LA negative, there could have been underdetection of LA). In only six out of the 34 discordant samples, LA status was tested on more than one occasion and consistent LA results were recorded (three as LA positive and three as negative) (Table 3). On this basis, it could be argued that the local/ hospital laboratory determination of LA might not be reliable in at least some of the $13 / 17$ discordant samples and 28/34 samples that gave equivocal results in the Core laboratories. This would potentially account for $80.4 \%$ (41/51) of samples where there was a disagreement in LA status between the Core and local/hospital laboratories, ie 41/166 (24.7\%) of non-anticoagulated samples. Results from the Core laboratories might also have contributed to the discordance, especially the ones judged equivocal, ie $20.5 \%$, $34 / 166$ of non-anticoagulated samples.

\section{4 | Anticoagulated patient samples}

All samples from anticoagulated patients were tested at a single Core laboratory (London, UK), which had the appropriate test repertoire available. The majority of patients receiving anticoagulants were on long-term warfarin or other VKA $(n=224)$, six were receiving the DOAC FXa inhibitor rivaroxaban, $18 \mathrm{LMWH}$, and four both 
TAB LE 4 Agreement in LA status between Core and local/hospital laboratories results for anticoagulated patient samples: The number of positive and negative LA samples identified by both laboratories and the ones that were discrepant are shown, as well as agreement and statistical results

\begin{tabular}{|c|c|c|c|c|c|c|}
\hline & & \multicolumn{5}{|c|}{ Core laboratory } \\
\hline & & POS & NEG & Agreement & K coeff & $G$-test ${ }^{a}$ \\
\hline \multirow[t]{2}{*}{ Local/hospital laboratories } & POS & 174 & 27 & $183 / 237$ & \multirow{2}{*}{$\begin{array}{l}.206 \\
P<.001\end{array}$} & \multirow{2}{*}{$\begin{array}{l}13.9 \\
\text { (99.98\% confidence) }\end{array}$} \\
\hline & NEG & 27 & 9 & $77.2 \%$ & & \\
\hline
\end{tabular}

Abbreviations: LA, lupus anticoagulant; NEG, negative; POS, positive.

${ }^{a}$ The $G$-test value indicates that there is $<0.02 \%$ chance of making a mistake regarding agreement of positive and negative LA results as defined in this study.

\begin{tabular}{|c|c|c|c|c|}
\hline \multirow{3}{*}{$\begin{array}{l}\text { Total } \\
\text { Local/hospital laboratories }\end{array}$} & \multicolumn{2}{|c|}{ Discordant } & \multicolumn{2}{|c|}{ Equivocal } \\
\hline & \multicolumn{2}{|l|}{54} & \multicolumn{2}{|l|}{15} \\
\hline & LA POS & LA NEG & LA POS & LA NEG \\
\hline $\begin{array}{l}\text { Samples tested on only one occasion at } \\
\text { local/hospital laboratory }\end{array}$ & 8 & 7 & 3 & 0 \\
\hline $\begin{array}{l}\text { Tested more than once at local/hospital } \\
\text { laboratory, but LA status varied }\end{array}$ & 9 & 11 & 1 & 3 \\
\hline $\begin{array}{l}\text { Method of LA assessment not specified or } \\
\text { only one LA method performed }\end{array}$ & 6 & 5 & 4 & 1 \\
\hline $\begin{array}{l}\text { Tested more than once at local/hospital } \\
\text { laboratory, with consistent results on LA } \\
\text { status }\end{array}$ & 4 & 4 & 2 & 1 \\
\hline
\end{tabular}

TAB LE 5 Analysis of possible reasons for discordant and equivocal results in LA status between Core and local/hospital laboratories testing in anticoagulated patient samples

Abbreviations: LA, lupus anticoagulant; NEG, negative; POS, positive.

VKA and LMWH. Of the 252 anticoagulated patient samples, 15 were considered to be equivocal by the Core laboratory, because they were negative by DRVVT and an inhibitor could not be demonstrated in the TVT. The remaining samples showed 77.2\% (183/237) agreement in LA classification. Discordance between the Core and local/hospital laboratories was identified in $22.7 \%$ (54/237) of samples with equal numbers of samples (27 each) identified as negative by either the Core or local/hospital laboratories and positive by the other (Table 4).

There was an additional $6.0 \%(15 / 252)$ of patients showing equivocal results; three were tested on only one occasion at the local/hospital laboratory; four were tested more than once, but with variable results; in five the LA methods were not specified or only one LA method was used; only three were tested more than once with consistent results (two LA positive, one negative) (Table 5).

We also examined possible reasons for the discordance in LA status between Core and local/hospital laboratories in the 54 anticoagulated samples, which were as follows: 15 were tested on only one occasion; 20 were tested more than once, but LA status varied; and in 11 the LA method was not specified or only one method was used. Only eight of the 54 samples were tested on more than one occasion with consistent results (four LA positive and four negative) (Table 5). Of the patients with discordant results, the LA method at the local/ hospital laboratory was recorded as "other" (than aPTT or DRVVT) in 10 cases, "aPTT-based" in five, as "unknown" in five, DRVVT in 14, and aPTT plus DRVVT in 20. On this basis, one could argue that the
LA status reported by the local/hospital laboratories might not be reliable in at least some of the $84 \%$ (58/69) samples with discordant or equivocal LA status (46/54 discordant and $12 / 15$ samples giving equivocal results), ie in 58/252 (23\%) of the overall anticoagulated patients. Results judged equivocal by the Core laboratories $(6.0 \%$, $15 / 252$ ) might also have contributed to the discordance observed with local/hospital laboratories in anticoagulated samples.

\section{4 | DISCUSSION}

In the first part of this prospective APS ACTION study, we demonstrated that good agreement and low variability in LA performance between the APS ACTION Core laboratories was achieved by use of the same reagents, analyzer type, and protocols. Furthermore, in the second part of the study we established that LA status results between Core and local/hospital laboratories were concordant in $87 \%$ (115/132 non-anticoagulated) and 77\% (anticoagulated) samples, when equivocal LA results were excluded. Importantly, however, we identified that $28.7 \%(120 / 418)$ of the total samples showed discordance or equivocal LA results between the Core and local/hospital laboratories. Thirteen percent (17/132) of non-anticoagulated and $22.7 \%$ (54/237) of anticoagulated patient samples were discordant between the two laboratories (when equivocal results were excluded). A further 20.5\% (34/166) and 6\% (15/252) of non-anticoagulated and anticoagulated patient samples, respectively, were 
identified as equivocal by the Core laboratories, but as positive or negative by the local/hospital laboratory. Further analysis indicated that the LA results in $80.4 \%$ (41/51) of non-anticoagulated and $84 \%$ $(58 / 69)$ of anticoagulated discordant/equivocal samples may not be reliable. This accounted for $24.7 \%$ (of 166 ) of the total non-anticoagulated and $23 \%$ (of 252) anticoagulated samples, respectively i.e. approximately $24 \%$ of all samples. These results indicate that it is important to use validated and standardized LA results, as provided by the Core laboratories, to underpin the accurate interpretation of APS ACTION clinical data.

There are many differences between haemostasis laboratories in the selection of LA tests, source of reagents, methodological detail, and results. ${ }^{2,13-16}$ External quality assessment studies in Europe have shown considerable variability between laboratories for LA detection, particularly in samples with "weak" LA, with false negative and false positive rates of $10 \%-20 \% .{ }^{17,18}$ In North America, false negative rates up to $28 \%$ and false positive rates of around $11 \%$ have been reported, while in Australasian studies, false negative rates up to $50 \%$ and false positive rates of about $10 \%$ were observed. ${ }^{14,19}$ These discrepancies appear to be due to a variety of pre- and post-analytical factors as well as performance of the tests. Within-method, but not betweenmethod, agreement could be improved by the use of common normal plasma for ratio calculations and the adoption of recommendations from national and international guidelines on LA tests. Between- and within-method agreement can also be improved by use of the mean reference range for clotting time for ratio calculation. ${ }^{13,18,19}$

The sensitivity and specificity of results also appears to depend on the potency of the LA and the method used. APTT-based methods generally appear to be more sensitive to weak and moderate potency LA than DRVVT methods, but have lower specificity. ${ }^{14}$ In one study, 29 different hospitals referred plasma samples from patients with LA to a reference laboratory, ${ }^{8}$ where they were retested using DRVVT and kaolin clotting time: $24 \%$ of samples were LA negative on retesting, either due to normal clotting times in screening tests, or complete correction in mixing tests to demonstrate an inhibitor. However, in this study, hospitals submitting samples had been asked to filter the plasma samples, which can introduce variability due to potential changes in clotting factor levels as well as inducing microparticle formation and consequent changes in the clotting time after freezing and thawing. ${ }^{20}$ Sample preparation is critical for LA detection, as platelet contamination of plasma samples can lead to false negative LA results after the plasma is frozen and stored. ${ }^{9}$ Local cut-off and reference ranges are important, as there are differences depending on the reagent and analyzer used ${ }^{21}$ as well as the method for calculating the values. ${ }^{22}$ In our study, following venipuncture, blood samples were double centrifuged to obtain PPP and stored at $-80^{\circ} \mathrm{C}$, in accordance with national and international guidelines. ${ }^{3-5}$

Notwithstanding the good agreement and precision in LA testing demonstrated between four Core Laboratories in the first part of our study, it is clearly important to monitor laboratory performance, particularly if laboratories are not experienced in performing haemostasis tests. One Core laboratory (whose expertise is in solid phase aPL and other immunoassays), produced markedly different results from the other four and therefore took no further part in LA assessment for APS ACTION. In hospital laboratories that regularly perform LA tests, such discrepant performance would be identified through external quality assessment participation schemes such as external quality assurance services (EQAS), United Kingdom National External Quality Assessment Service (UK NEQAS), External quality Control of diagnostic Assays and Tests (ECAT foundation).

There are a variety of possible reasons for the observed discordance between Core and local/hospital laboratories in the second part of the study. Laboratories implement the various guidance documents $^{3-5}$ for LA detection in different ways and adherence is influenced by the type of automation available, local analyzer protocols, laboratory computer systems, and the economics of batch testing compared to single sample analysis. The guidelines recommend the performance of two different LA tests employing different methodologies, only one of which needs to indicate LA. However, in some laboratories, only one test system is available. This is a particular problem in samples from anticoagulated patients, where some test systems (eg aPTT-based tests) may not be appropriate due to their sensitivity to warfarin, ${ }^{23}$ or where a heparin-neutralizing agent is not included in the reagent. According to APS criteria, if LA positivity is the sole laboratory criteria for diagnosis, it should be demonstrated on at least two occasions 12 weeks or more apart. ${ }^{1}$ Some local/hospital laboratories contributing to APS ACTION only tested for LA on one occasion while they confirmed persistence of aCL/a $\beta 2 \mathrm{GPI}$, but not LA, more than once; other laboratories only used a single LA reagent; and some obtained variable results on different blood samples, meaning that LA may have been present but undetected in some patients. Our data suggesting that at least some of the local/ hospital LA results may not be reliable in up to $24.7 \%$ of the total non-anticoagulated and $23 \%$ of the total anticoagulated samples, is of particular clinical importance as the result could have a major impact on patient management. Core laboratory results were limited because samples could only be tested at a single time point (and in some cases with different reagents and methods to local/hospital laboratories) and this could have introduced differences and contributed to the discordance with the local/hospital LA results. This is particularly critical for equivocal samples $(20.5 \%$ and $6 \%$ of nonanticoagulated and anticoagulated samples, respectively) and these need later re-evaluation; longitudinal testing of registry samples collected in subsequent years will enable determination of the LA status of all patients in the APS ACTION registry. However, the purpose of the Core laboratories testing was to provide a highly standardized, consistent assessment of LA status.

International APS criteria and LA testing guidance also state that part of LA testing should be to demonstrate an inhibitor, usually achieved by testing mixtures of patient and normal plasmas. ${ }^{3-5}$ The results for some samples were deemed to be equivocal in the Core laboratories, because a prolonged clotting time could not be demonstrated in plasma mixtures. However, this does not prove that they are LA negative, as aPL may have been diluted in the mixing test to below the threshold for detection; nor does a prolonged screening test but normal mixing test mean that the patient definitely has a 
"weak" LA, as the clotting time could have been prolonged for other reasons.

Lupus anticoagulant assessment in samples from patients receiving VKA anticoagulation is particularly challenging, because most LA tests require the presence of vitamin $K$ dependent coagulation factors. As also concluded in our study, the discordance in LA status between the local/hospital and Core laboratories was more prevalent in the anticoagulated samples $(22.7 \%, 54 / 237)$ when compared to non-anticoagulated ones $(12.9 \%, 17 / 132)(P=.03)$. LA testing in anticoagulated samples is generally discouraged as the probability for false positive/negative results is high. ${ }^{24,25}$ However, determination of LA status is required in certain clinical settings as well as for full characterization of aPL status of patients in research studies and registries such as APS ACTION. Where the INR is $<4.0$, it is generally accepted that DRVVT Screen and Confirm procedures can be used on equal volume mixtures of patient and control plasma (with evidence of an inhibitor provided where the DRVVT Screen normalized ratio is still abnormal). ${ }^{26}$ APTT-based tests, including SCT, can be problematic, due to their vitamin $\mathrm{K}$ factor sensitivity and great prolongation of clotting time. Textarin or Taipan venoms, which are direct activators of prothrombin and are little affected by VKA, can be used, although textarin venom is currently difficult to obtain. Coupled with Ecarin venom, the TVT/ECT ratio is useful for LA testing in VKA anticoagulated patients. ${ }^{27-30}$ The TVT/ECT ratio is less sensitive than the DRVVT, and thus a positive result may be useful, but a negative result should be considered as "not detected." Taipan venom also shows low sensitivity to heparin pentasaccharide, although in the presence of long chain heparin antithrombin can inhibit the taipan venom by a template-dependent mechanism similar to that of thrombin inhibition. ${ }^{31}$ There is an even greater problem with samples from patients receiving DOACs. In our study, only six patients were receiving rivaroxaban; however, the number of patients receiving DOACs may increase in APS ACTION in future years. FXa inhibitors interfere with aPTT-based tests and DRVVT and may thus result in erroneous results. It has been suggested that the DRVVT can be used in the drug trough period, however, false positive results have been reported even at very low ( $<50 \mathrm{ng} / \mathrm{mL}$ ) rivaroxaban levels. ${ }^{32}$ We assessed samples containing rivaroxaban using the TVT/ECT, which is not affected by rivaroxaban. ${ }^{33,34}$ Both the TVT and ECT tests were performed with patient plasma rather than equal volume mixtures of patient and normal plasma, as the venom reagents lead to direct activation of factor II and show little effect of VKA and direct FXa inhibitor anticoagulation. However, a mixing test was performed for TVT in this study if the TVT clotting time was prolonged and if sufficient plasma was available, to establish the presence of an inhibitor. Limitations to this approach were that: only one LA test (TVT/ECT) was available for samples from DOAC-treated patients and performing a single test might increase the risk of false-negative results; samples were tested on one occasion only; and the sensitivity of the TVT/ECT is generally less than that for DRVVT. ${ }^{35}$ This problem may be resolved in the future by the use of DOAC removal agents, which are showing encouraging results, ${ }^{36,37}$ and with testing of longitudinal samples. The use of cut-offs for undiluted plasma for interpretation of the mixing tests is also a limiting factor in this study as this might lead to reduced detection rates and could have increased the numbers of equivocal and false-negative results.

\section{5 | CONCLUSIONS}

Good agreement in LA performance can be achieved, as in the APS ACTION Core Laboratories, by use of the same reagents, analyzer type, and protocols. The observed agreement in LA test results (positive/negative) between Core and local/hospital laboratories of $87 \%$ non-anticoagulated samples and $77 \%$ anticoagulated samples (when equivocal results were excluded), appeared acceptable on initial review, especially when considering high interlaboratory variability, and the difficulties of detecting LA in samples from anticoagulated patients as well as lack of adherence to guidelines. However, 28.7\% (120/418) of samples showed discordance between the Core and local/hospital laboratories, or equivocal LA results. Analysis of possible reasons for discordance/equivocal LA suggested that at least some of the results of the local/hospital laboratories might not have been reliable in $24.7 \%(41 / 166)$ and $23 \%(58 / 252)$ of the total non-anticoagulated and anticoagulated samples, respectively. Results by the Core laboratories judged as equivocal might also have contributed in the discordance in $20.5 \%$ (34/166) and 6\% (15/252) of non-anticoagulated and anticoagulated samples, respectively. Thus, standardized Core laboratory LA testing is required to underpin the accurate interpretation of APS ACTION clinical data. Studies on LA testing on the annual follow-up samples from the APS ACTION registry patients will enable future re-evaluation of longitudinal LA data and enable correct identification and validation of LA status. Our study has highlighted the need for widespread standardization of LA testing, to underpin accurate diagnosis and management of APS patients.

\section{ACKNOWLEDGMENTS}

We would like to thank NIBSC, South Mimms, UK for kindly providing the 1st International Reference Panel for LA plasma; Werfen for providing the ACL TOP500 analyzer and LA reagents; and Diagnostic Reagents Ltd for providing Taipan and Ecarin venoms; without having any input in the study and/or analysis of the data or results. Data management was performed using REDCAP provided by the Clinical and Translational Science Center at Weill Cornell Medicine (CTSC grant UL1 TR000457).

\section{CONFLICT OF INTEREST}

The authors declare that they have no relevant conflict of interest.

\section{AUTHOR CONTRIBUTIONS}

Maria Efthymiou designed and carried out the study, analyzed data, performed statistical analysis, and wrote the first draft of the 
manuscript. Ian J. Mackie designed the study, analyzed data, and reviewed the manuscript. Philip J. Lane performed experiments, data handling, and reviewed the manuscript. Hannah Cohen designed the study and provided critical revision of the manuscript. Vittorio Pengo, Danieli Andrade, Rohan Willis, Steve Krilis, and Laura M. Bertolaccini contributed to writing the laboratory manual for Core laboratories and oversaw the laboratory work at their sites. Doruk Erkan was involved in designing the study and led the overall APS ACTION work. Savino Sciascia performed statistical analysis, Elisa Bison, Margarete Borges Galhardo Vendramini, Zurina Romay-Penabad, Miao Qi, performed experiments at the respective Core laboratory. Joann Vega and Ecem Sevim performed research duties including patient recruitment and data monitoring and maintenance of the APS ACTION database. Amaia Ugarte, Cecilia B. Chighizola, H. Michael Belmont, Lanlan Ji, M $^{a}$ Angeles Aguirre Zamorano, Guilherme de Jesus, D. Ware Branch, Paul R. Fortin, Laura Andreoli, Michelle Petri, Esther Rodriguez, Ricard Cervera, Jason S. Knight, and Tatsuya Atsumi were involved in patient recruitment and data entry into the APS ACTION database and registry. All authors contributed to revising the intellectual content of the manuscript and final approval of the version to be published.

\section{ORCID}

Maria Efthymiou iD https://orcid.org/0000-0001-9704-2845

Ian J. Mackie iD https://orcid.org/0000-0002-7393-2365

Philip J. Lane iD https://orcid.org/0000-0001-9335-1819

Rohan Willis (iD https://orcid.org/0000-0002-1374-7678

Doruk Erkan iD https://orcid.org/0000-0001-7216-677X

Savino Sciascia (iD https://orcid.org/0000-0003-1266-9441

Maria Tektonidou (iD https://orcid.org/0000-0003-2238-0975

Ricard Cervera iD https://orcid.org/0000-0001-6085-492X

Jason S. Knight (iD https://orcid.org/0000-0003-0995-9771

Tatsuya Atsumi (iD https://orcid.org/0000-0001-5657-962X

Ecem Sevim (iD https://orcid.org/0000-0001-6458-9844

Maria Laura Bertolaccini (iD https://orcid.org/0000-0002-8623-139X

Vittorio Pengo (iD https://orcid.org/0000-0003-2064-6071

Hannah Cohen iD https://orcid.org/0000-0003-2032-390X

\section{REFERENCES}

1. Miyakis S, Lockshin MD, Atsumi T, et al. International consensus statement on an update of the classification criteria for definite antiphospholipid syndrome (APS). J Thromb Haemost. 2006;4:295-306.

2. Tripodi A. Laboratory testing for lupus anticoagulants: a review of issues affecting results. Clin Chem. 2007;53:1629-1635.

3. Pengo V, Tripodi A, Reber G, et al. Update of the guidelines for lupus anticoagulant detection. Subcommittee on Lupus Anticoagulant/ Antiphospholipid Antibody of the Scientific and Standardisation Committee of the International Society on Thrombosis and Haemostasis. J Thromb Haemost. 2009;7:1737-1740.
4. Keeling D, Mackie I, Moore GW, Greer IA, Greaves M. Guidelines on the investigation and management of antiphospholipid syndrome. Br J Haematol. 2012;157:47-58.

5. CLSI Guideline. Laboratory Testing for the Lupus Anticoagulant; Approved Guideline. CLSI document H60-A, 2014. 2014. Clinical and Laboratory Standards Institute, Wayne, PA, USA.

6. Erkan D, Lockshin MD. APS ACTION-AntiPhospholipid Syndrome Alliance For Clinical Trials and InternatiOnal Networking. Lupus. 2012;21:695-698.

7. CLSI Guidline. Defining, Establishing, and Verifying Reference Intervals in the Clinical Laboratory; Approved Guideline. CLSI document EP28-A3c. 2008. 2008. Clinical and Laboratory Standard Institute, Wayne, PA, USA.

8. Pengo V, Biasiolo A, Gresele P, et al. Survey of lupus anticoagulant diagnosis by central evaluation of positive plasma samples. $J$ Thromb Haemost. 2007;5:925-930.

9. Odsaeter IH, Lian IA, Bratberg K, Mikkelsen G. Dry ice exposure of plasma samples influences $\mathrm{pH}$ and lupus anticoagulant analysis. Clin Chem Lab Med. 2015;53:809-813.

10. Arachchillage DR, Mackie IJ, Efthymiou M, Isenberg DA, Machin $\mathrm{SJ}$, Cohen $\mathrm{H}$. Interactions between rivaroxaban and antiphospholipid antibodies in thrombotic antiphospholipid syndrome. J Thromb Haemost. 2015;13:1264-1273.

11. Cohen J. Weighted kappa: nominal scale agreement with provision for scaled disagreement or partial credit. Psychol Bull. 1968;70:213-220

12. Xu S, Lorber MF. Interrater agreement statistics with skewed data: evaluation of alternatives to Cohen's kappa. J Consult Clin Psychol. 2014;82:1219-1227.

13. Adams M. Measurement of lupus anticoagulants: an update on quality in laboratory testing. Semin Thromb Hemost. 2013;39:267-271.

14. Dembitzer FR, Ledford Kraemer MR, Meijer P, Peerschke El. Lupus anticoagulant testing: performance and practices by North American clinical laboratories. Am J Clin Pathol. 2010;134: 764-773.

15. Devreese K, Hoylaerts MF. Laboratory diagnosis of the antiphospholipid syndrome: a plethora of obstacles to overcome. Eur J Haematol. 2009;83:1-16.

16. Moffat KA, Ledford-Kraemer MR, Plumhoff EA, et al. Are laboratories following published recommendations for lupus anticoagulant testing? An international evaluation of practices. Thromb Haemost. 2009;101:178-184.

17. Jennings I, Kitchen S, Woods TA, Preston FE, Greaves M. Potentially clinically important inaccuracies in testing for the lupus anticoagulant: an analysis of results from three surveys of the UK National External Quality Assessment Scheme (NEQAS) for Blood Coagulation. Thromb Haemost. 1997;77:934-937.

18. Jennings I, Greaves M, Mackie IJ, Kitchen S, Woods TA, Preston FE. Lupus anticoagulant testing: improvements in performance in a UK NEQAS proficiency testing exercise after dissemination of national guidelines on laboratory methods. $\mathrm{Br} J$ Haematol. 2002;119:364-369.

19. Favaloro EJ, Bonar R, Marsden K. Internal quality control and external quality assurance in testing for antiphospholipid antibodies: part II - lupus anticoagulant. Semin Thromb Hemost. 2012;38:404-411.

20. Favaloro EJ. Preanalytical variables in coagulation testing. Blood Coagul Fibrinolysis. 2007;18:86-89.

21. Gardiner C, Mackie IJ, Malia RG, et al. The importance of locally derived reference ranges and standardized calculation of dilute Russell's viper venom time results in screening for lupus anticoagulant. Br J Haematol. 2000;111:1230-1235.

22. Tripodi A, Chantarangkul V, Cini M, et al. Variability of cut-off values for the detection of lupus anticoagulants: results of an international multicenter multiplatform study. J Thromb Haemost. 2017:15:1180-1190. 
23. Dembitzer FR, Suarez Y, Aledort LM, Peerschke El. Screening coagulation testing using the APTT: which reagent to choose? Am J Hematol. 2010;85:726.

24. Aboud M, Morel-Kopp MC, Ward C, Coyle L. False-negative or false-positive: laboratory diagnosis of lupus anticoagulant at the time of commencement of anticoagulant. J Thromb Haemost. 2010;8:2070-2073.

25. Tripodi A, Moia M, Pengo V. False-negative or false-positive: laboratory diagnosis of lupus anticoagulant at the time of commencement of anticoagulant: a rebuttal. J Thromb Haemost. 2011;9: 1435-1436.

26. Pengo V, Tripodi A, Reber G, et al. Update of the guidelines for lupus anticoagulant detection. Subcommittee on Lupus Anticoagulant/ Antiphospholipid Antibody of the Scientific and Standardisation Committee of the International Society on Thrombosis and Haemostasis. J Thromb Haemost. 2009;7:1737-1740.

27. Rooney AM, McNally T, Mackie IJ, Machin SJ. The Taipan snake venom time: a new test for lupus anticoagulant. J Clin Pathol. 1994;47:497-501.

28. Parmar K, Lefkou E, Doughty H, Connor P, Hunt BJ. The utility of the Taipan snake venom assay in assessing lupus anticoagulant status in individuals receiving or not receiving an oral vitamin $\mathrm{K}$ antagonist. Blood Coagul Fibrinolysis. 2009;20:271-275.

29. Moore GW, Smith MP, Savidge GF. The Ecarin time is an improved confirmatory test for the Taipan snake venom time in warfarinized patients with lupus anticoagulants. Blood Coagul Fibrinolysis. 2003;14:307-312

30. Triplett DA, Stocker KF, Unger GA. Barna LK. The Textarin/Ecarin ratio: a confirmatory test for lupus anticoagulants. Thromb Haemost. 1993;70:925-931.

31. Chen L, Rezaie AR. Proexosite-1-dependent recognition and activation of prothrombin by taipan venom. J Biol Chem. 2004;279:17869-17874.

32. Martinuzzo ME, Forastiero R, Duboscq C, et al. False-positive lupus anticoagulant results by DRVVT in the presence of rivaroxaban even at low plasma concentrations. Int J Lab Hematol. 2018;40:e99-e101.
33. Arachchillage DR, Efthymiou M, Mackie IJ, Lawrie AS, Machin SJ, Cohen H. Rivaroxaban and warfarin achieve effective anticoagulation, as assessed by inhibition of TG and in-vivo markers of coagulation activation, in patients with venous thromboembolism. Thromb Res. 2015;135:388-393.

34. van Os GM, de LB, Kamphuisen PW, Meijers JC, de Groot PG. Detection of lupus anticoagulant in the presence of rivaroxaban using Taipan snake venom time. J Thromb Haemost. 2011;9:1657-1659.

35. Moore GW, Culhane AP, Maloney JC, Archer RA, Breen KA, Hunt BJ. Taipan snake venom time coupled with ecarin time enhances lupus anticoagulant detection in nonanticoagulated patients. Blood Coagul Fibrinolysis. 2016;27:477-480.

36. Favresse J, Lardinois B, Sabor L, et al. Evaluation of the DOAC-Stop procedure to overcome the effect of DOACs on several thrombophilia screening tests. Thromb Haemost. 2018;2:e202-e209.

37. Exner T, Michalopoulos N, Pearce J, Xavier R, Ahuja M. Simple method for removing DOACs from plasma samples. Thromb Res. 2018;163:117-122.

\section{SUPPORTING INFORMATION}

Additional supporting information may be found online in the Supporting Information section at the end of the article.

How to cite this article: Efthymiou M, Mackie IJ, Lane PJ, et al. Comparison of real world and core laboratory lupus anticoagulant results from the Antiphospholipid Syndrome Alliance for Clinical Trials and International Networking (APS ACTION) clinical database and repository. J Thromb Haemost. 2019;17:2069-2080. https://doi.org/10.1111/jth.14596 